International Journal of Pure and Applied Mathematics

Volume 111 No. 3 2016, 373-381

ISSN: 1311-8080 (printed version); ISSN: 1314-3395 (on-line version)

url: http://www.ijpam.eu

doi: 10.12732 /ijpam.v111i3.2

\title{
ON SOME APPLICATIONS OF THE MULTI-DIMENSIONAL NEW FRACTIONAL CALCULUS FOR THE RIEMANN-LIOUVILLE WITH ATANGANA-BALEANU
}

\author{
Adem Kılıçman ${ }^{1}$, Wasan Ajeel Ahmood ${ }^{2} \S$ \\ ${ }^{1,2}$ Department of Mathematics and \\ nstitute for Mathematical Research \\ University Putra Malaysia \\ 43400 UPM, Serdang, Selangor, MALAYSIA \\ ${ }^{2}$ Department of Al-Quran Science \\ Faculty of Education for Women
Al-Iraqia University
Baghdad, IRAQ

\begin{abstract}
In this paper, we presented new fractional derivative including Riemann-Liouville with Laplace transform has a non-local and non-singular kernel was proposed by Atangana and Baleanu and extended to fractional partial derivative with multi-dimensional Laplace transform also presented new fractional integral. The aim from this topic to answer some outstanding questions that were posed by many researchers within the field of fractional calculus. The results are presented and we obtain a chaotic behavior which was not obtained by local derivative.
\end{abstract}

AMS Subject Classification: 26A33, 44A10

Key Words: Atangana-Baleanu derivatives, integral and Laplace transform

\section{Introduction}

Integral transforms are applied to solve new fractional derivative and integral

Received:

Revised: April 6, 2016

Published: June 29, 2016

$\S$ Correspondence author (c) 2016 Academic Publications, Ltd. url: www.acadpubl.eu 
including Riemann-Liouville proposed by Atangana and Baleanu. Recently some new integral transforms were introduced, see [1] and applied to solve some ODE as well as PDEs and they gave the relation between both derivatives with Laplace, Sumudu, Fourier and Mellin transform for the AtanganaBaleanu fractional derivative in Caputo sense and RiemannLiouville sense.

Many researchers (see [1],..,[10]) have great success by their derivative have no singular kernel and find applications in many real world problems and applying them in the field of groundwater and thermal science. Caputo and Fabrizio obtained the results from the version based upon the Riemann-Liouville approach was developed by Atangana with Goufo also, presented many theoretical works (see [11], [12]).

A issues was pointed out in Riemann-Liouville sense, the kernel was not nonlocal, the integral associate is not a fractional operator but the average of the function and its integral, and the solution of the following equation $\frac{d^{\alpha} y}{d x^{\alpha}}=a y$ is exponential equation not a non-local function.

Therefore, some researchers concluded that the fractional parameter can then be view as filter regulator. In the order, Riemann-Liouville has a big problem that, his kernel although non-local but is singular. This weakness has effect when modeling real world problems.

\section{Basic Definitions of New Fractional Derivative with Basic Method}

We start this section by the following basic definitions of the new derivatives with fractional order proposed by Atangana and Baleanu. Also, in detail some new properties and theorems.

The following definition gives the definition of the Riemann-Liouville fractional derivative.

Definition 1 (New Riemann-Liouville Fractional Derivative, see [13). ] Let $f \in H^{1}(a, b), b>a, \alpha \in[0,1]$ and not necessary differentiable then, the definition of the AtanganaBaleanu fractional derivative in RiemannLiouville sense is given as:

$$
{ }_{b}^{A B R} D_{x}^{\alpha}(f(x))=\frac{B(\alpha)}{1-\alpha} \frac{d}{d x} \int_{b}^{x} f(t) E_{\alpha}\left[-\alpha \frac{(x-T)^{\alpha}}{1-\alpha}\right] d t .
$$

The above definition will be helpful to real world problem and also will have great advantage when using Laplace transform to solve some physical problem with initial condition. 
Definition 2. The fractional integral associate to the new fractional derivative with non-local kernel is defined as:

$$
{ }_{a}^{A B} I_{x}^{\alpha}(f(x))=\frac{1-\alpha}{B(\alpha)} f(x)+\frac{\alpha}{B(\alpha) \Gamma(\alpha)} \int_{a}^{x} f(t)(x-t)^{\alpha-1} d t .
$$

When alpha is zero we recover the initial function and if also alpha is 1 , we obtain the ordinary integral.

\section{Solution of the Linear Ordinary New Riemann-Liouville Fractional Derivative Via the Laplace Transform, see [13]}

Consider Fractional order for one-dimensional new Riemann-Liouville fractional derivative

$$
{ }_{0}^{A B R} D_{x}^{\alpha}(f(x))=\frac{B(\alpha)}{1-\alpha} \frac{d}{d x} \int_{0}^{x} f(t) E_{\alpha}\left[-\alpha \frac{(x-T)^{\alpha}}{1-\alpha}\right] d t .
$$

Then, by taking the Laplace transform of the above equation, we have:

$$
\begin{aligned}
L\left\{{ }_{0}^{A B R} D_{x}^{\alpha}(f(x))\right\}(s) & =L\left\{\frac{B(\alpha)}{1-\alpha} \frac{d}{d x} \int_{0}^{x} f(t) E_{\alpha}\left[-\alpha \frac{(x-T)^{\alpha}}{1-\alpha}\right] d t\right\} \\
& =\frac{B(\alpha)}{1-\alpha} \frac{s^{\alpha} L\{f(x)\}(s)}{s^{\alpha}+\frac{\alpha}{1-\alpha}}
\end{aligned}
$$

To illustrate this method, consider the following examples, see [13]:

1. When $f(x)=\frac{}{1} \frac{1}{\pi x}-\frac{2 a}{\pi} e^{-a^{2} x} \int_{0}^{a^{\sqrt{x}}} e^{\tau^{2}} d \tau$, then:

$L\left\{{ }_{0}^{A B R} D_{x}^{\alpha}\left(\frac{1}{\sqrt{\pi x}}-\frac{2 a}{\sqrt{\pi}} e^{-a^{2} x} \int_{0}^{a^{\sqrt{x}}} e^{\tau^{2}} d \tau\right)\right\}(s)=\frac{B(\alpha)}{1-\alpha} \frac{s^{\alpha}\left\{\frac{{ }^{\sqrt{s}}}{s+a^{2}}\right\}(s)}{s^{\alpha}+\frac{\alpha}{1-\alpha}}$

2. When $f(x)=t^{\beta-1} E_{\alpha, \beta}\left(a t^{\alpha}\right)$, then:

$$
L\left\{{ }_{0}^{A B R} D_{x}^{\alpha}\left(x^{\beta-1} E_{\alpha, \beta}\left(a x^{\alpha}\right)\right)\right\}(s)=\frac{B(\alpha)}{1-\alpha} \frac{s^{\alpha}\left\{\frac{s^{\alpha-\beta}}{s^{\alpha}-a}\right\}(s)}{s^{\alpha}+\frac{\alpha}{1-\alpha}} .
$$


Now, we present integral of some especial function in this section:

3. When $f(x)=E_{\alpha}\left(x^{\alpha}\right)$, then:

From definition (2)

$$
{ }_{0}^{A B} I_{x}^{\alpha}\left(E_{\alpha}\left(x^{\alpha}\right)\right)=\frac{1-\alpha}{B(\alpha)} E_{\alpha}\left(x^{\alpha}\right)+\frac{\alpha}{B(\alpha) \Gamma(\alpha)} \int_{0}^{x} E_{\alpha}\left(t^{\alpha}\right)(x-t)^{\alpha-1} d t .
$$

Where

$$
\frac{1}{\Gamma(\alpha)} \int_{0}^{x} E_{\alpha}\left(t^{\alpha}\right)(x-t)^{\alpha-1} d t=E_{\alpha}\left(x^{\alpha}\right)-1
$$

Put this equation and replacing in the above fractional integral equation, we obtain:

$$
{ }_{0}^{A B} I_{x}^{\alpha}\left(E_{\alpha}\left(x^{\alpha}\right)\right)=\frac{1}{B(\alpha)} E_{\alpha}\left(x^{\alpha}\right)-\frac{\alpha}{B(\alpha)} .
$$

4. When $f(x)=x^{\beta-1} E_{\alpha, \beta}\left(\sigma x^{\alpha}\right)$, then from definition (2), we have

$$
\begin{aligned}
{ }_{0}^{A B} I_{x}^{\alpha}\left(x^{\beta-1} E_{\alpha, \beta}\left(\sigma x^{\alpha}\right)\right)=\frac{1-\alpha}{B(\alpha)} & x^{\beta-1} E_{\alpha, \beta}\left(\sigma x^{\alpha}\right) \\
& +\frac{\alpha}{B(\alpha) \Gamma(\alpha)} \int_{0}^{x} t^{\beta-1} E_{\alpha, \beta}\left(\sigma t^{\alpha}\right)(x-t)^{\alpha-1} d t .
\end{aligned}
$$

However,

$$
\frac{\alpha}{B(\alpha) \Gamma(\alpha)} \int_{0}^{x} t^{\beta-1} E_{\alpha, \beta}\left(\sigma t^{\alpha}\right)(x-t)^{\alpha-1} d t=\frac{\alpha}{B(\alpha)} x^{\beta+\alpha-1} E_{\alpha, \beta+\alpha}\left(\sigma x^{\alpha}\right) .
$$

\section{The Multi-Dimensional Laplace Transform with Some Applications}

Definition 3 (New Multi-Dimensional Riemann-Liouville Fractional Derivative). Let $f \in H^{1}(a, b), b>a, \alpha \in[0,1]$ then, the definition of the new multidimensional Riemann-Liouville fractional derivative is given as:

$$
{ }_{b}^{A B R} D_{x}^{\alpha}\left(f\left(x_{1}, x_{2}, \ldots, x_{n}\right)\right)=\prod_{i=1}^{n}\left\{\frac{B\left(\alpha_{i}\right)}{1-\alpha_{i}}\right\} \frac{\partial^{\sum_{i=1}^{n} i}}{\partial x_{1} \partial x_{2} \ldots \partial x_{n}} \int_{b_{1}}^{x_{1}} \int_{b_{2}}^{x_{2}} \ldots \int_{b_{n}}^{x_{n}} f\left(t_{1}, t_{2}, \ldots, t_{n}\right)
$$




$$
\prod_{i=1}^{n} E_{\alpha_{i}}\left[-\alpha_{i} \frac{\left(x_{i}-T_{i}\right)^{\alpha_{i}}}{1-\alpha_{i}}\right] d t_{1} d t_{2} \ldots d t_{n}
$$

Then, by taking the multi-dimensional Laplace transform to both sides of the above equation, we have:

$$
\begin{aligned}
L_{n}\left\{\begin{array}{l}
A B R \\
0
\end{array} D_{x}^{\alpha}\left(f\left(x_{1}, x_{2}, \ldots, x_{n}\right)\right)\right\}\left(s_{1}, s_{2}, \ldots, s_{n}\right) \\
\quad=\prod_{i=1}^{n}\left\{\frac{B\left(\alpha_{i}\right)}{1-\alpha_{i}}\right\} \frac{\prod_{i=1}^{n} s_{i}^{\alpha_{i}} F\left(s_{1}, s_{2}, \ldots, s_{n}\right)}{\prod_{i=1}^{n}\left(s_{i}^{\alpha_{i}}+\frac{\alpha_{i}}{1-\alpha_{i}}\right)} .
\end{aligned}
$$

To illustrate this method, consider the following examples.

1. When

$$
\begin{aligned}
& f\left(x_{1}, x_{2}, \ldots, x_{n}\right) \\
& =\prod_{i=1}^{n}\left(\frac{2}{a_{i} \sqrt{\pi}} e^{-a_{i}^{2} x_{i}}\right) \int_{0}^{a_{1}{ }^{\sqrt{x_{1}}} a_{2}} \int_{0}^{\sqrt{x_{2}}} \ldots \int_{0}^{a_{n}{ }^{\sqrt{x_{n}}}} e^{-\sum_{i=1}^{n} a_{i}^{2} \tau_{i}^{2}} d \tau_{1} d \tau_{2} \ldots d \tau_{n},
\end{aligned}
$$

then:

$$
\begin{aligned}
L_{n}\left\{\begin{array} { r } 
{ A B R } \\
{ 0 }
\end{array} D _ { x } ^ { \alpha } \left(\prod_{i=1}^{n}\left(\frac{2}{a_{i} \sqrt{\pi}} e^{-a_{i}^{2} x_{i}}\right)\right.\right. \\
\left.\quad \int_{0}^{\left.a_{1}{ }^{\sqrt{ } \overline{x_{1}} a_{2} \overline{x_{2}}} \int_{0}^{a_{n}{ }^{\overline{x_{n}}}} e^{-\sum_{i=1}^{n} a_{i}^{2} \tau_{i}^{2}} d \tau_{1} d \tau_{2} \ldots d \tau_{n}\right)}\right\}\left(s_{1}, s_{2}, \ldots, s_{n}\right) \\
=\prod_{i=1}^{n}\left\{\frac{B\left(\alpha_{i}\right)}{1-\alpha_{i}}\right\} \frac{\prod_{i=1}^{n} s_{i}^{\alpha_{i}} \prod_{i=1}^{n}\left(\frac{1}{\bar{s}_{i}\left(s_{i}+a_{i}^{2}\right)}\right)}{\prod_{i=1}^{n}\left(s_{i}^{\alpha_{i}}+\frac{\alpha_{i}}{1-\alpha_{i}}\right)} .
\end{aligned}
$$

2. When $f\left(x_{1}, x_{2}, \ldots, x_{n}\right)=\prod_{i=1}^{n}\left(x_{i}^{\alpha_{i}} E_{1, \alpha_{i}+1}\left(a_{i} x_{i}\right)\right)$, then:

$$
\begin{aligned}
& L_{n}\left\{\begin{array}{l}
A B R \\
0
\end{array} D_{x}^{\alpha}\left(\prod_{i=1}^{n}\left(x_{i}^{\alpha_{i}} E_{1, \alpha_{i}+1}\left(a_{i} x_{i}\right)\right)\left(s_{1}, s_{2}, \ldots, s_{n}\right)\right)\right\} \\
= & \prod_{i=1}^{n}\left\{\frac{B\left(\alpha_{i}\right)}{1-\alpha_{i}}\right\} \frac{\prod_{i=1}^{n}\left(\frac{1}{s_{i}^{\alpha_{i}}\left(s_{i}-a_{i}\right)}\right)}{\prod_{i=1}^{n}\left(s_{i}^{\alpha_{i}}+\frac{\alpha_{i}}{1-\alpha_{i}}\right)}
\end{aligned}
$$


3. When $f\left(x_{1}, x_{2}, \ldots, x_{n}\right)=\prod_{i=1}^{n}\left(x_{i}^{\beta_{i}-1} E_{\alpha_{i}, \beta_{i}}\left(a_{i} x_{i}^{\alpha_{i}}\right)\right)$, then:

$L_{n}\left\{{ }_{0}^{A B R} D_{x}^{\alpha}\left(\prod_{i=1}^{n}\left(x_{i}^{\beta_{i}-1} E_{\alpha_{i}, \beta_{i}}\left(a_{i} x_{i}^{\alpha_{i}}\right)\right)\right)\left(s_{1}, s_{2}, \ldots, s_{n}\right)\right\}=\prod_{i=1}^{n}\left(\frac{s_{i}^{\alpha_{i}-\beta_{i}}}{s_{i}^{\alpha_{i}}-a_{i}}\right)$.

Definition 4 (New Riemann Liouville Multi-Dimensional Fractional Integral). Let $\alpha_{i}$ for each $i=1,2, \ldots, n$ be a real non-negative number. Let $\mathrm{f}$ be a function of $x_{1}, x_{2}, \ldots, x_{n}$ integrable on any finite subinterval of $J=[0, \infty]$. Then, for $x_{i}>0$ for each $i=1,2, \ldots, n$, is the new Riemann-Liouville fractional integral of $\mathrm{f}$ of order $\alpha_{i}$ for each $i=1,2, \ldots, n$.

$$
\begin{aligned}
\prod_{i=1}^{n} I_{x_{i}}^{\alpha_{i}}= & \prod_{i=1}^{n}\left\{\frac{1-\alpha_{i}}{B\left(\alpha_{i}\right)}\right\} f\left(x_{1}, x_{2}, \ldots, x_{n}\right) \\
& +\prod_{i=1}^{n}\left\{\frac{\alpha_{i}}{B\left(\alpha_{i}\right) \Gamma\left(\alpha_{i}\right)}\right\} \int_{a_{n}}^{x_{n}} \int_{a_{n-1}}^{x_{n-1}} \ldots \int_{a_{1}}^{x_{1}} \prod_{i=1}^{n}\left(x_{i}-t_{i}\right)^{\alpha_{i}-1} \\
& f\left(t_{1}, t_{2}, \ldots, t_{n}\right) d t_{1} d t_{2} \ldots d t_{n}, \alpha_{i}>0, \quad i=0,1,2, \ldots, n
\end{aligned}
$$

Where $\mathrm{f}$ is a known function of $x_{1}, x_{2}, \ldots, x_{n}$.

Now, we present multi-dimensional integral of some function:

4. When $f\left(x_{1}, x_{2}, x_{3}\right)=\frac{1}{\frac{1}{2} ! \frac{5}{2} ! \frac{7}{3} !}\left(x_{1}^{\frac{1}{2}} x_{2}^{\frac{5}{2}} x_{3}^{\frac{7}{3}}\right)+x_{1}^{\frac{1}{4}} x_{2}^{\frac{3}{4}} x_{3}^{\frac{5}{4}}$, then:

From Definition 3:

$$
\begin{aligned}
\prod_{i=1}^{3} I_{x_{i}}^{\alpha_{i}}= & \prod_{i=1}^{3}\left\{\frac{1-\alpha_{i}}{B\left(\alpha_{i}\right)}\right\}\left\{\frac{1}{\frac{1}{2} ! \frac{5}{2} ! \frac{7}{3} !}\left(x_{1}^{\frac{1}{2}} x_{2}^{\frac{5}{2}} x_{3}^{\frac{7}{3}}\right)+x_{1}^{\frac{1}{4}} x_{2}^{\frac{3}{4}} x_{3}^{\frac{5}{4}}\right\} \\
& +\prod_{i=1}^{3}\left\{\frac{\alpha_{i}}{B\left(\alpha_{i}\right) \Gamma\left(\alpha_{i}\right)}\right\} \int_{0}^{x_{3}} \int_{0}^{x_{2}} \int_{0}^{x_{1}} \prod_{i=1}^{3}\left(x_{i}-t_{i}\right)^{\alpha_{i}-1} \\
& \left(\frac{1}{\frac{1}{2} ! \frac{5}{2} ! \frac{7}{3} !}\left(t_{1}^{\frac{1}{2}} t_{2}^{\frac{5}{2}} t_{3}^{\frac{7}{3}}\right)+t_{1}^{\frac{1}{4}} t_{2}^{\frac{3}{4}} t_{3}^{\frac{5}{4}}\right) d t_{1} d t_{2} d t_{3}, \alpha_{i}>0, \quad i=0,1,2,3
\end{aligned}
$$

where

$$
\begin{gathered}
\frac{1}{\prod_{i=1}^{3} \Gamma\left(\alpha_{i}\right)} \int_{0}^{x_{3}} \int_{0}^{x_{2}} \int_{0}^{x_{1}} \prod_{i=1}^{3}\left(x_{i}-t_{i}\right)^{\alpha_{i}-1}\left(\frac{1}{\frac{1}{2} ! \frac{5}{2} ! \frac{7}{3} !}\left(t_{1}^{\frac{1}{2}} t_{2}^{\frac{5}{2}} t_{3}^{\frac{7}{3}}\right)+t_{1}^{\frac{1}{4}} t_{2}^{\frac{3}{4}} t_{3}^{\frac{5}{4}}\right) d t_{1} d t_{2} d t_{3} \\
=\frac{x_{1}^{\alpha_{1}+\frac{3}{2}-1} x_{2}^{\alpha_{2}+\frac{7}{2}-1} x_{3}^{\alpha_{3}+\frac{10}{3}-1}}{\Gamma\left(\alpha_{1}+\frac{3}{2}\right) \Gamma\left(\alpha_{2}+\frac{7}{2}\right) \Gamma\left(\alpha_{3}+\frac{10}{3}\right)}+\frac{x_{1}^{\alpha_{1}+\frac{5}{4}-1} x_{2}^{\alpha_{2}+\frac{7}{4}-1} x_{3}^{\alpha_{3}+\frac{9}{4}-1}}{\Gamma\left(\alpha_{1}+\frac{5}{4}\right) \Gamma\left(\alpha_{2}+\frac{7}{4}\right) \Gamma\left(\alpha_{3}+\frac{9}{4}\right)}
\end{gathered}
$$


Replacing in the above equation, we obtain:

$$
\begin{aligned}
\prod_{i=1}^{3} I_{x_{i}}^{\alpha_{i}}= & \prod_{i=1}^{3}\left\{\frac{1-\alpha_{i}}{B\left(\alpha_{i}\right)}\right\}\left\{\frac{1}{\frac{1}{2} ! \frac{5}{2} ! \frac{7}{3} !}\left(x_{1}^{\frac{1}{2}} x_{2}^{\frac{5}{2}} x_{3}^{\frac{7}{3}}\right)+x_{1}^{\frac{1}{4}} x_{2}^{\frac{3}{4}} x_{3}^{\frac{5}{4}}\right\}+\prod_{i=1}^{3}\left\{\frac{\alpha_{i}}{B\left(\alpha_{i}\right)}\right\} \\
& \frac{x_{1}^{\alpha_{1}+\frac{3}{2}-1} x_{2}^{\alpha_{2}+\frac{7}{2}-1} x_{3}^{\alpha_{3}+\frac{10}{3}-1}}{\Gamma\left(\alpha_{1}+\frac{3}{2}\right) \Gamma\left(\alpha_{2}+\frac{7}{2}\right) \Gamma\left(\alpha_{3}+\frac{10}{3}\right)}+\frac{x_{1}^{\alpha_{1}+\frac{5}{4}-1} x_{2}^{\alpha_{2}+\frac{7}{4}-1} x_{3}^{\alpha_{3}+\frac{9}{4}-1}}{\Gamma\left(\alpha_{1}+\frac{5}{4}\right) \Gamma\left(\alpha_{2}+\frac{7}{4}\right) \Gamma\left(\alpha_{3}+\frac{9}{4}\right)}
\end{aligned}
$$

5. When $f\left(x_{1}, x_{2}\right)=e^{a\left(x_{1}\right)+b\left(x_{2}\right)}$, then: From definition (3)

$$
\begin{aligned}
\prod_{i=1}^{2} I_{x_{i}}^{\alpha_{i}}= & \prod_{i=1}^{2}\left\{\frac{1-\alpha_{i}}{B\left(\alpha_{i}\right)}\right\}\left\{e^{a\left(x_{1}\right)+b\left(x_{2}\right)}\right\} \\
& +\prod_{i=1}^{2}\left\{\frac{\alpha_{i}}{B\left(\alpha_{i}\right) \Gamma\left(\alpha_{i}\right)}\right\} \int_{0}^{x_{2}} \int_{0}^{x_{1}} \prod_{i=1}^{2}\left(x_{i}-t_{i}\right)^{\alpha_{i}-1} \\
& \left(e^{a\left(t_{1}\right)+b\left(t_{2}\right)}\right) d t_{1} d t_{2}, \alpha_{i}>0, i=0,1,2
\end{aligned}
$$

where

$$
\begin{array}{r}
\frac{1}{\prod_{i=1}^{2} \Gamma\left(\alpha_{i}\right)} \int_{0}^{x_{2}} \int_{0}^{x_{1}} \prod_{i=1}^{2}\left(x_{i}-t_{i}\right)^{\alpha_{i}-1}\left(e^{a\left(t_{1}\right)+b\left(t_{2}\right)}\right) d t_{1} d t_{2} \\
=x^{\alpha_{1}} E_{1,\left(\alpha_{1}+1\right)}(a x) \cdot y^{\alpha_{2}} E_{1,\left(\alpha_{2}+1\right)}(b y) .
\end{array}
$$

Replacing in the above equation, we obtain:

$$
\begin{aligned}
\prod_{i=1}^{2} I_{x_{i}}^{\alpha_{i}}=\prod_{i=1}^{2}\left\{\frac{1-\alpha_{i}}{B\left(\alpha_{i}\right)}\right\} & \left\{e^{a\left(x_{1}\right)+b\left(x_{2}\right)}\right\} \\
& +\prod_{i=1}^{2}\left\{\frac{\alpha_{i}}{B\left(\alpha_{i}\right)}\right\} x^{\alpha_{1}} E_{1,\left(\alpha_{1}+1\right)}(a x) \cdot y^{\alpha_{2}} E_{1,\left(\alpha_{2}+1\right)}(b y)
\end{aligned}
$$

\section{Conclusion}

In this work, we proposed (M.D.L.T.M) for solving multi-dimensional new fractional calculus for the Riemann-Liouville with Atangana-Baleanu. It is illustrated that, this method is power and active tool to find the solution of new 
fractional linear partial differential equations and multi-dimensional integral equations. Furthermore, the M.D.L.T.M has been successfully applied to nd an exact solution of fractional partial differential equations, to solve for the transform of the unknown function and applied to nd solution of fractional multi-dimensional integral equations.

\section{References}

[1] A. Atangana, On the new fractional derivative and application to nonlinear Fisher's reaction-diffusion equation, Appl Math Comput. (2016), 948-56.

[2] H. Jordan, Transient heat diffusion with a non-singular fading memory: from the cattaneo constitutive equation with Jeffrey's kernel to the Caputo-Fabrizio time-fractional derivative, Therm Sci. (2016).

[3] D. Goufo, M. Pene, N. Jeanine, Duplication in a model of rock fracture with fractional derivative without singular kernel, Open Math. (2015), 839-46.

[4] J.Gomez-Aguilar, H. Yepez-Martinez, C. Caldern-Ramon, I. Cruz-Orduna, R. EscobarJimenez, R. Escobar-Jimenez, V. Olivares-peregrinoictor, Modeling of a mass-springdamper system by fractional derivatives with and without a singular kernel, Entropy (2015), 6289-303.

[5] D. Bizezinski, Accuracy problem of numerical calculation of fractional order derivatives and integrals applying the Riemann-Liouville/Caputo formulas, Appl Math Nonlinear Sci., 1 (2016), 23-43.

[6] J. Jiang, D. Cao, H. Chen, Boundary value problems for fractional differential equation with causal operators, Appl. Math Nonlinear Sci., 1 (2016), 23-43.

[7] S. Kumar, A new analytical modeling for telegraph equation via Laplace transform, Appl. Math Model, 38 (13) (2014), 3154-63.

[8] S. Kumar, M. Rashdi, New analytical method forgas dynamics equation arising in shock froms, Comput phys Commun, 285 (7) (2014), 1947-54.

[9] S. Kumar, J. Yao, A. Kumar, A fractional model to describing the Brownian motion of particles and its analytical solution, Adv Mech Eng., 7 (12) (2015), 1-11.

[10] S. Kumar, X. Yin, D. Kumar, A modified homotopy analysis method for solution of fractional wave equations, Adv Mech Eng., 7 (12) (2015), 1-8.

[11] M. Caputo, M. Fabrizio, Applications of new time and spatial fractional derivatives with exponential kernels, Progr. Fract. Differ. Appl., 2 (2016), 1-11.

[12] A. Alsaedi, D. Baleanu, S. Etemad, S. Rezapour, On coupled systems of time-fractional differential problems by using a new fractional derivative, J. Funct Space (2016), 8.

[13] A. Atangana, K. Ilknur, Chaos in a simple nonlinear system with AtanganaBaleanu derivatives with fractional order, Chaos, Solitons and Fractals (2016), 1-8. 